\title{
Germline epigenetics, and reprogramming in zebrafish early embryos
}

\author{
Magdalena E Potok, David A Nix, Timothy J Parnell, Bradley R Cairns ${ }^{*}$ \\ From Epigenetics and Chromatin: Interactions and processes \\ Boston, MA, USA. 11-13 March 2013
}

Early vertebrate embryos must achieve totipotency and prepare for zygotic genome activation (ZGA). To better understand, we determined DNAme profiles of zebrafish gametes, multiple embryo stages flanking ZGA, and somatic muscle - and compared them to gene activity and histone modifications. First, sperm chromatin patterns are virtually identical to those at ZGA. Unexpectedly, in the oocyte many genes important for germline functions (ie. piwil1) or early development (ie. hox genes) are DNA methylated - yet demethylated during zygotic/cleavage stages to precisely the state observed in sperm. Remarkably, this cohort constitutes the genes/loci that acquire DNAme during development (ie. ZGA to muscle). Furthermore, DNA methyltransferase inhibition experiments suggests that DNAme silences particular gene/chromatin cohorts at ZGA, preventing their precocious expression. Thus, zebrafish appear to achieve a 'totipotent' chromatin state at ZGA through paternal genome competency, maternal genome DNA demethylation/reprogramming, and the imposition of DNA methylation on genes needed later in development.

Submit your next manuscript to BioMed Central and take full advantage of:

- Convenient online submission

- Thorough peer review

- No space constraints or color figure charges

- Immediate publication on acceptance

- Inclusion in PubMed, CAS, Scopus and Google Scholar

- Research which is freely available for redistribution 